\title{
Preliminary analysis of double-plateau ground motions of recent great earthquakes
}

\author{
Yinfeng Dong ${ }^{a}$, Ying Hu and Song Lin \\ Key Laboratory of New Technology for Construction of Cities in Mountain Area \\ (Chongqing University), Ministry of Education, Chongqing, 400045, China, \\ School of Civil Engineering, Chongqing University, Chongqing, 400045, China
}

\begin{abstract}
A large number of ground motions were obtained during recent great earthquakes such as the 2008 Wenchuan earthquake and 2011 Tohoku earthquake and some of them present double-plateau features, this paper focuses on the identification and spatial distribution of double-plateau ground motions. First, the energy-time distribution of ground motions are described by a piece-wised envelope function with double plateau, which is proposed in previous study. Then, the envelope parameters of 10 ground motions with double plateau and 10 ground motions with single plateau are used as training sample, those ground motions are classified into two groups by support vector machine, i.e., single- and double-plateau. Finally, the spatial distribution of double-plateau ground motions along the direction of ruptures are analyzed and lay the foundation for further study.
\end{abstract}

Keywords: earthquake, ground motion, spatial distribution, plateau, envelope function.

\section{Introduction}

The 2008 Wenchuan earthquake and the 2011 Tohoku earthquake caused serious casualties, property loss and damage to the environment. The 2008 Wenchuan earthquake left more than 87,000 dead and missing, and around 82,000 people were died and injured during the 2011 Tohoku earthquake. Because of the high density distribution of high precision digital accelerographs, a large number of acceleration records in high quality were obtained, analyses conducted on those records and showed that there were a considerable number of ground motions with an obvious double-plateau feature in time history curves during those two great earthquakes. This phenomenon is due to large zone, long duration of rupture process, multiple rupture, huge energy release and Doppler effect[1-3], and has never been so clearly revealed in records from previous earthquakes. Peng[4] found that the spatial distribution of this kind of ground motions is directional and is consistent with the distribution of region with serious damage after he analyzed the data of the Tohoku earthquake. While it needs to be further investigated that whether this laws is universal for different earthquakes or not. Furthermore, it has been concerned that there are differences between conventional ground motions with single plateau and ground motions with double plateau in the engineering characteristics and the effects on structure.

\footnotetext{
${ }^{a}$ Corresponding author : dongyinfeng@cqu.edu.cn
} 


\section{The envelope function model of double-plateau ground motions}

In previous studies, an envelope function model with double plateau was proposed [3](as shown in Fig. 1)

$$
\begin{gathered}
E(t)=\sqrt{E_{1}(t)^{2}+E_{2}(t)^{2}} \\
E_{1}(t)= \begin{cases}0 & t \leq t_{01} \\
I_{01}\left(\frac{t-t_{01}}{t_{11}-t_{01}}\right)^{2} & t_{01}<t \leq t_{11} \\
I_{01} & t_{11}<t \leq t_{21} \\
I_{01} e^{-c_{1}\left(t-t_{21}\right)} & t>t_{21}\end{cases} \\
E_{1}(t)= \begin{cases}0 & t \leq t_{01} \\
I_{01}\left(\frac{t-t_{01}}{t_{11}-t_{01}}\right)^{2} & t_{01}<t \leq t_{11} \\
I_{01} & t_{11}<t \leq t_{21} \\
I_{01} e^{-c_{1}\left(t-t_{21}\right)} & t>t_{21}\end{cases}
\end{gathered}
$$

Eqs. (1)-(3) and Fig. 1 show that the envelop function $E(t)$ consists of two conventional singleplateau envelop functions, $E_{1}(\mathrm{t})$ and $E_{2}(\mathrm{t})$. This envelop function can describe the energy-time distribution feature of ground motions with single or double plateau and consists of 10 parameters, 6 time parameters $t_{01}, t_{11}, t_{21}, t_{02}, t_{12}, t_{22}$. 2 intensity parameters $I_{01}$ and $I_{02}$ for the plateau section; 2 attenuation indexes $c_{1}$ and $c_{2}$ for the downward section. Furthermore, these time parameters satisfy the following inequality

$$
t_{01} \leq t_{11} \leq t_{21} \leq t_{12} \leq t_{22}
$$

According to the above function model, it is easy to get the normalized accumulated energy (Husid) $h(t)$

$$
\begin{gathered}
h(t)=C \cdot h_{1}(t)+(1-C) \cdot h_{2}(t) \\
C=I_{01}^{2} /\left(I_{01}^{2}+I_{02}^{2}\right) \\
h_{1}(t)= \begin{cases}0 & t \leq t_{01} \\
\frac{1}{5}\left(t-t_{01}\right)^{5} /\left(t_{11}-t_{01}\right)^{4} & t_{01}<t \leq t_{11} \\
\frac{t-\frac{4}{5} t_{11}-\frac{1}{5} t_{01}}{h_{0}^{1}} & t_{11}<t \leq t_{21} \\
\frac{e^{-2 c_{1}\left(t-t_{21}\right)}}{h_{0}^{1}} & t>t_{21}\end{cases}
\end{gathered}
$$




$$
\begin{aligned}
& h_{0}^{1}=t_{21}-\frac{4}{5} t_{11}-\frac{1}{5} t_{01}+\frac{1}{2 c_{1}} \\
& h_{2}(t)=\left\{\begin{array}{lc}
0 & t \leq t_{02} \\
\frac{\frac{1}{5}\left(t-t_{02}\right)^{5} /\left(t_{12}-t_{02}\right)^{4}}{h_{0}^{2}} & t_{02}<t \leq t_{12} \\
\frac{t-\frac{4}{5} t_{12}-\frac{1}{5} t_{02}}{h_{0}^{2}} & t_{12}<t \leq t_{22} \\
1-\frac{e^{-2 c_{2}\left(t-t_{22}\right)}}{h_{0}^{2}} & t>t_{22}
\end{array}\right. \\
& h_{0}^{2}=t_{22}-\frac{4}{5} t_{12}-\frac{1}{5} t_{02}+\frac{1}{2 c_{2}}
\end{aligned}
$$

Eq. (5)-(10) shows that the total normalized accumulated energy of the model is the superposition of the energies of the two single-plateau envelop functions, and the respective proportions of accumulated energy of those two envelop functions is $C$ and (1-C).

According to the normalized accumulated energy model as Eq. (5) shows, the actual normalized accumulated energy function can be taken as the objective function to determine model parameters above, by using optimization method. Intensity parameters $I_{01}$ and $I_{02}$ can be obtained according to the total energy of the earthquake ground motions and Eq. (6).

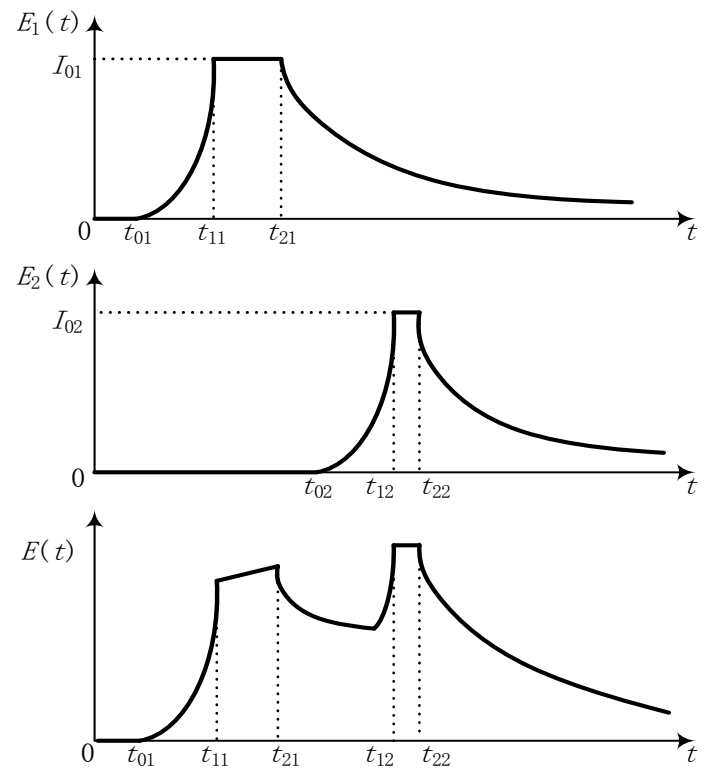

Figure 1. Envelope model with double plateau.

Fig.2 shows the intensity envelope curves and fitted curves of Husid by analyzing the east-west direction component of strong motions records, which obtained at the IWT008 station during the Tohoku earthquake. The values of 9 envelope parameters $t_{01}, t_{11}, t_{21}, c_{1}, C, t_{02}, t_{12}, t_{22}$ and $c_{2}$ were got as $22.13 \mathrm{~s}, 30.78 \mathrm{~s}, 32.84 \mathrm{~s}, 0.064,0.492,54.43 \mathrm{~s}, 71.60 \mathrm{~s}, 79.50 \mathrm{~s}$ and 0.032 , it is obvious that those ground motions have double plateau. 


\section{The identification of double-plateau ground motions}

Support vector machine (SVM), proposed by Vapnik et al in 1992, is an efficient data classification method which has been well developed in recent years[5]. Because of its unique theoretical background and classification method, this method can solve many problems that can't be solved by other classification algorithms. Support vector machine is built on Structural Risk Minimum principle and VC (Vapnik-Chervonenkis) dimension theory of statistic 1earning theory and its essence is a convex quadratic programming problem in mathematical programming. For convex quadratic programming problem in mathematical programming, the local optimal solution is the global optimal solution, which provides support vector machine with a strong generalization ability.
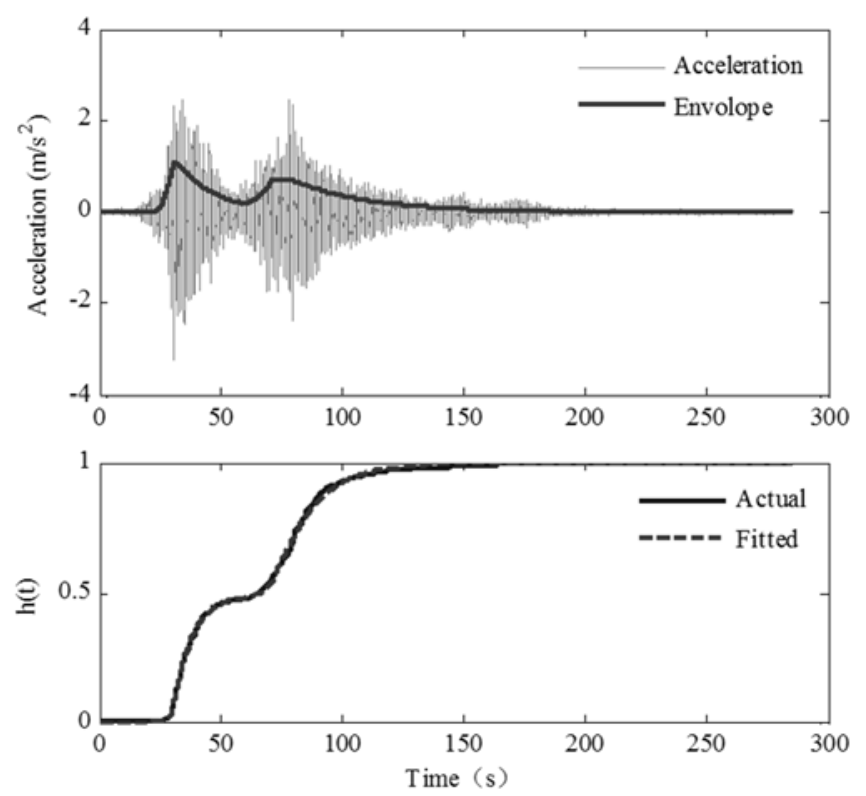

Figure 2. Envelope and Husid curve of strong ground motion recorded at station IWT008 ( EW component).

At present, SVM has been successfully applied in the field of data classification, and has achieved good results. Researchers proposed many different classification methods of SVM for different research fields and purposes. In this paper, data training method was used and this method has its specific process, including[5-6] training samples determination, feature extraction generation, SVM training, data classification(regression analysis). The principle of SVM is that samples to be classified are mapped into high dimensional feature space by nonlinear mapping (eigenvectors), and the optimal classification hyper plane is created in this space.

Considering the advantages of SVM in data classification, this paper used 9 intensity envelope parameters to construct training samples, then the SVM after training was used to divide ground motions into two types, those with single plateau features and double plateau features. Considering the record lengths from different stations are different, 6 time parameters were normalized when constructing samples, which means the ratios of those 6 time parameters to record durations were used to construct training samples.

In this paper, 10 single-plateau records and 10 double-plateau records were chosen randomly. Two horizontal acceleration components and a vertical acceleration component from those records were used to synthesize a vector, after that the module of the vector was used to calculate the normalized accumulated energy $h(t)$, which was used to identify those envelope parameters, then those parameters were used to construct training data. After the training of SVM, all the records can be classified on the basis of envelope parameters. 


\section{The distribution of double-plateau ground motions}

According to the classification results, statistical analyses were conducted on the fault distance, peak acceleration, spatial distribution characteristics for those two types of records.

Fig. 3 shows the statistical distribution of fault distances in the two earthquakes. It can be seen that in the Whenchuan earthquake, the double-plateau ground motions are mainly distributed near the fault zone while the single-plateau ground motions are distributed relatively far away from the fault zone. However, in the Tohoku earthquake, the two types of ground motions show same distribution pattern with respect to fault distances. This is mainly because that the Wenchuan earthquake occurred in inland and strong earthquake records were obtained on both sides of faults, while the Tohoku earthquake struck off Japan's eastern coast and strong earthquake records were only obtained from the inland side (hanging wall side).

Fig. 4 and Fig. 5 show the distribution of the peak ground acceleration (PGA) for the ground motions with single or double plateau from the two great earthquakes, which is similar to the distribution of the fault distances. For the Whenchuan earthquake, the PGA for double-plateau ground motions are mainly distributed within the range of 60-120gal, and the PGA for single-plateau are mainly distributed below 60 gal, while above the range of 120 gal, the distribution ratio of the ground motions with double plateau is obviously larger than that with double plateau. In the records of the Tohoku earthquake, however, the distributions of PGA with single or double plateau are basically similar and most data are below 180 gal.

Fig. 6 shows the spatial distributions of strong motions records with single or double plateau from the two great earthquakes. For the Whenchuan earthquake, the double-plateau records are mainly distributed faults and are mainly correspond to the epicentral area, while single-plateau records are mainly distributed far away from faults and out of the epicentral area. Also, there are laws for the distribution of the double-plateau records along the direction of ruptures, that is, along the positive direction of main ruptures, the number of double-plateau records is obviously lower than that along the opposite direction. For the Tohoku earthquake, the spatial distribution laws of the double-plateau records are more obvious, and the recording stations are mainly distributed in an area which is in the opposite direction to the main ruptures.

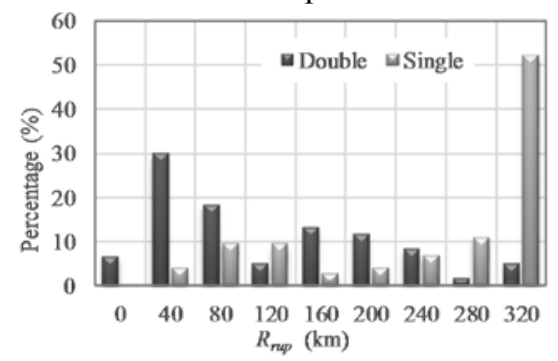

(a) Wenchuan earthquake

Figure 3. Rupture distances of strong motion records.

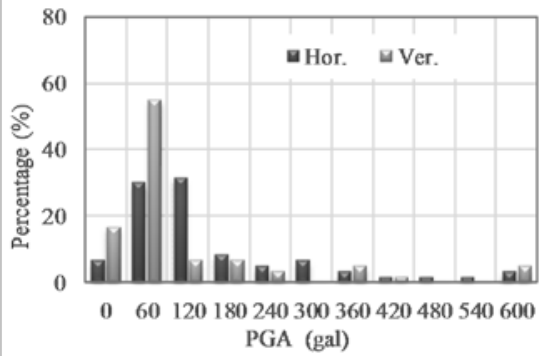

(a)Double-plateau

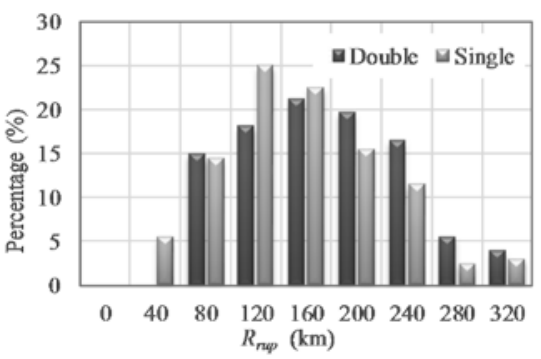

(b)Tohoku earthquake

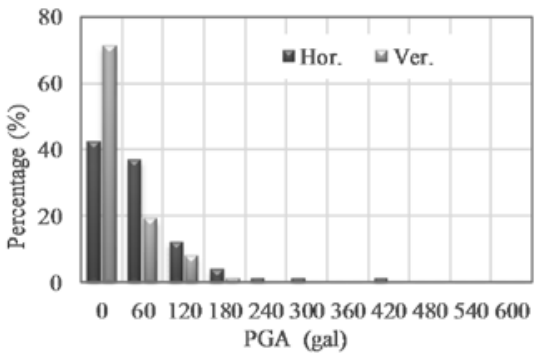

(b)Single-plateau

Figure 4. Peak ground acceleration of strong motion records from Wenchuan earthquake. 


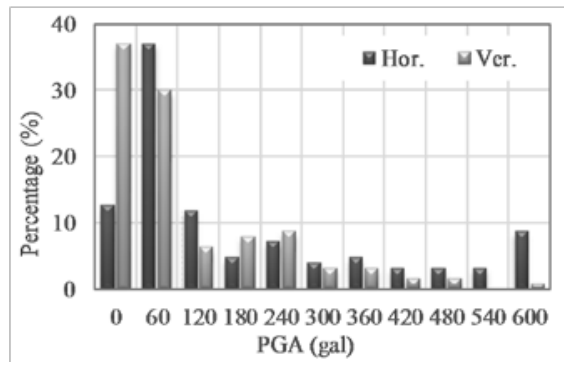

(a)Double-plateau

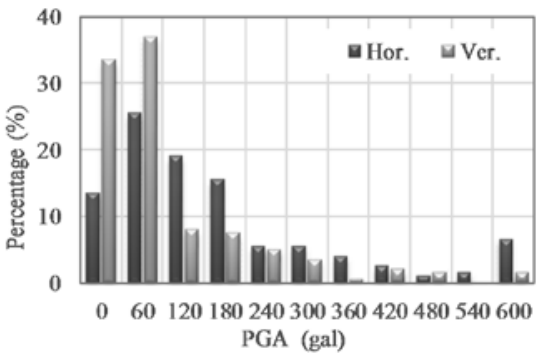

(b)Single-plateau

Figure 5. Peak ground acceleration of strong motion records from Tohoku earthquake.

To better illustrate the relationship between the rupture process of the faults and the distribution areas of double-plateau records, Fig. 7 shows the acceleration time history curves obtained from stations parallel to faults(west east component). It can be seen that the distribution laws of the doubleplateau records from the two great earthquakes are similar to Fig. 6. The spatial propagation laws of seismic waves generated by multiple (more than two) fault ruptures can be clearly distinguished by analyzing the chronological order of plateau peaks. The time intervals for main ruptures of the Wenchuan earthquake are relatively short, while in the Tohoku earthquake, the intervals for main ruptures are rather long and the duration of ruptures are also longer.
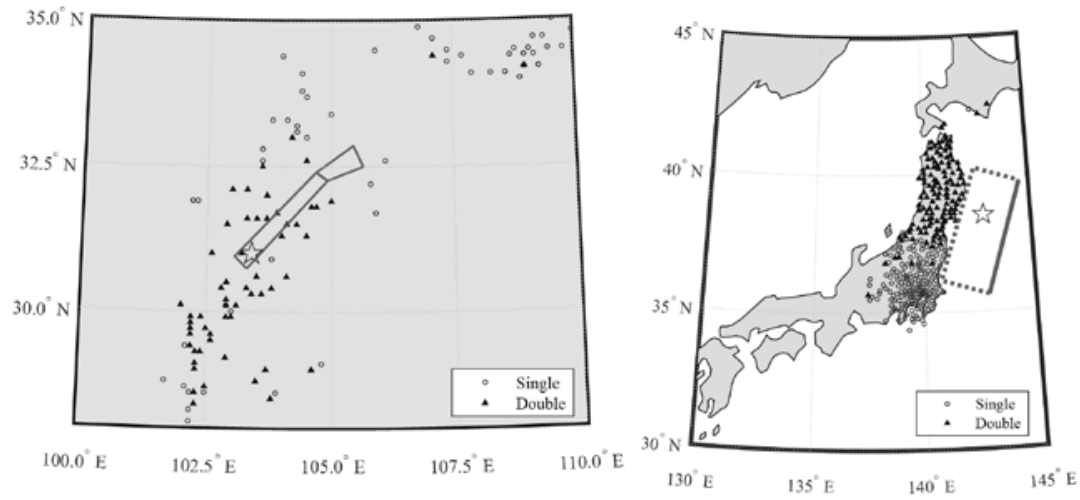

(a) Wenchuan earthquake(b)Tohoku earthquake

Figure 6. Spatial distribution of strong motions with single or double plateau from Wenchuan and Tohoku earthquakes.

\section{Conclusions}

In this paper, an envelope function with double plateau was used to describe the energy-time distribution of ground motions firstly. Then, support vector machine was used to classify and extract ground motions with double plateau. Finally, the spatial distribution of double-plateau ground motions along the direction of ruptures was explored. It is found that double-plateau ground motions are the consequences resulting from multiple rupture processes. Double-plateau ground motions are generally distributed along the opposite direction of the main ruptures. The time intervals of plateau peaks are closely related to the time intervals of fault ruptures, and the quantitative relationship between those two kinds of intervals will be one of the contents of the follow-up study. 

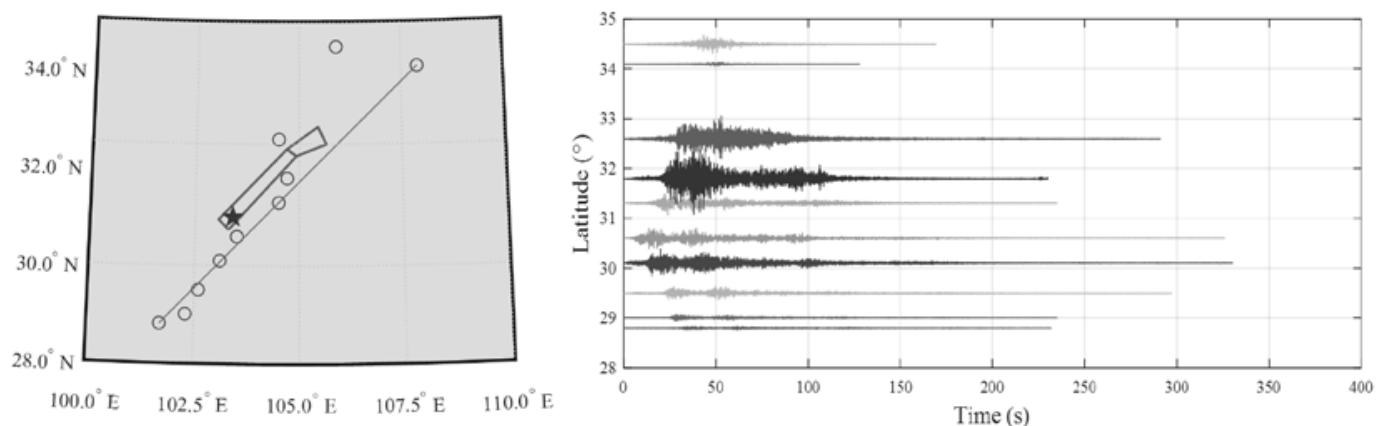

(a) Wenchuan earthquake
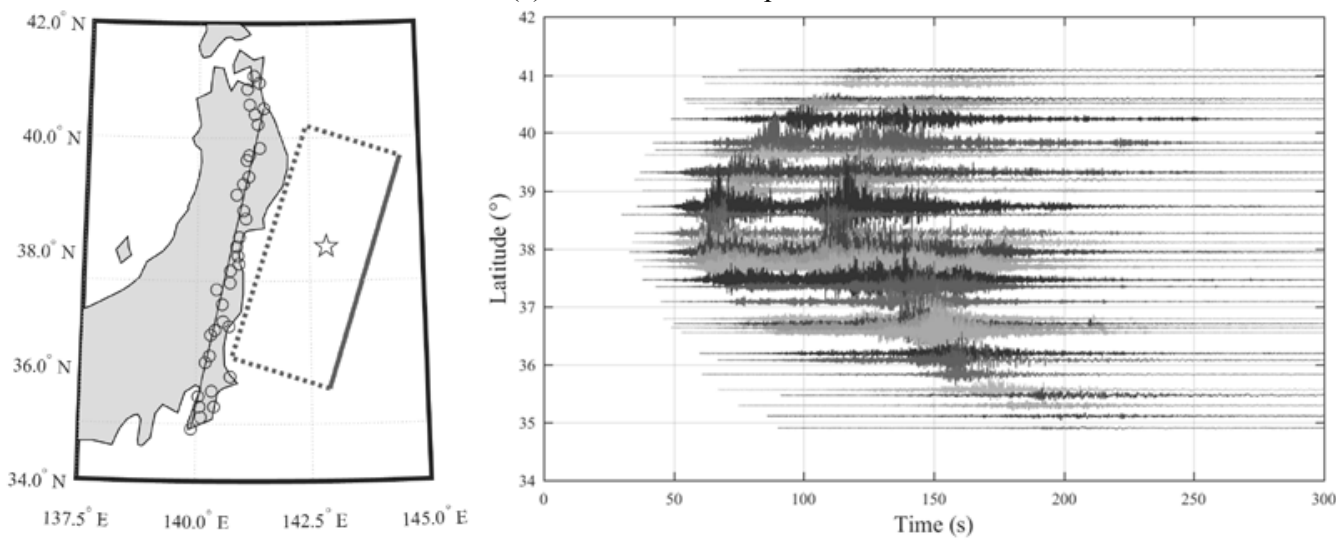

(b)Tohoku earthquake

Figure 7. Acceleration time history of strong motions recorded at stations parallel to faults for Wenchuan and Tohoku earthquakes.

\section{Acknowledgments}

This work is supported by National Natural Science Foundation of China under Grant No. 10802104..

\section{References}

1. Maercklin N, Gaetano F, Colombelli S, et al. Twin ruptures grew to build up the giant 2011 Tohoku, Japan, earthquake. Scientific Reports, 2012, 709(2):1-7.

2. Wang WM, Zhao LF, Li J, et al. Rupture process of the Ms 8.0 Wenchuan earthquake of Sichuan, China. Chinese Journal of Geophysics, 2008, 51(5): 1403-1410 (in Chinese).

3. Yan K M. Engineering Properties of Strong Ground Motions with Double-plateau in Envelope, Master's thesis, Chongqing University(2015) (in Chinese).

4. Peng B. Engineering Properties of Strong Ground Motions from Seismic Sequences of the2011Tohoku Earthquake, Master's thesis, Chongqing University(2013)(in Chinese).

5. Vapnik V. The nature of statistical learning theory. (New York: Springer, 1995).

6. Liang Y. The Research on the Extension and Application of SVM Classification, Master's thesis, Hunan University(2008)(in Chinese). 